\title{
Analysis on the Riding Characteristics of Mobike Sharing Bicycle in Beijing City, China
}

\author{
Min $\mathrm{Cao}^{\mathrm{a}, \mathrm{b}, \mathrm{c}, *}$, Mengxue Huang ${ }^{\mathrm{a}, \mathrm{b}, \mathrm{c}}$ \\ ${ }^{a}$ Key Lab of Virtual Geographic Environment(Nanjing Normal University), Ministry of Education, Nanjing, 210023, China; \\ ${ }^{b}$ State Key Laboratory Cultivation Base of Geographical Environment Evolution (Jiangsu Province), Nanjing, 210023, China \\ c Jiangsu Center for Collaborative Innovation in Geographical Information Resource Development and Application, Nanjing, \\ 210023, China
}

* Corresponding author

Keywords: Dock-less shared bicycle, Matrix of riding pattern, Riding characteristic, Beijing City

The development of the sharing economy has provided an important realization path for urban's green and healthy development, and has also accelerated the speed of urban development. With the constant capital pouring into the public transport field, dock-less shared bicycle is a relatively new form of transport in urban areas, and it provides a bikesharing service to fulfil urban short trips. Dock-less shared bicycle, with a characteristic of riding and stopping anywhere, has successfully solved the last mile travel problem. Recently, studies focus on the on the temporal spatial characteristics of public bicycle based on public bicycle operation data. However, there are few studies on the identification of riding patterns based on the characteristics of temporal and spatial behavior of residents. In addition, researches have been conducted on public bicycles administered by the government, and the dock-less shared bicycle have different characteristics from public bicycles in terms of scale of use and mode of use. This paper aims to analyze the temporal and spatial characteristics of residents using shared bicycles, and attempts to explore the characteristics of the riding modes of the dock-less shared bicycles.

Mobike sharing bicycle dataset of Beijing city were obtained for the research and this dataset contains a wealth of attributes with cover of 396600 shared bicycle users and 485500 riding records from May 10 to May 25 in 2017. Additionally, 19 types of POI (Point of Interest) data were also obtained through the API of Baidu Maps. To examine the patterns of shared bicycle trips, these POI data are categorized into five types including residential, commercial, institution, recreation and transport. Spatiotemporal analysis method, correlation analysis methods and kernel density methods were used to analyse the temporal and spatial characteristics of shared bicycle trips, revealing the time curve and spatial hotspot distribution area of shared bikes. Furthermore, a new matrix of riding pattern based on POI was proposed to identify the riding patterns during massive sharing bicycle dataset.

This paper aims to explore the riding behaviour of shared bicycles, and the research results are as follows:

(1) Temporal characteristics of riding behaviour

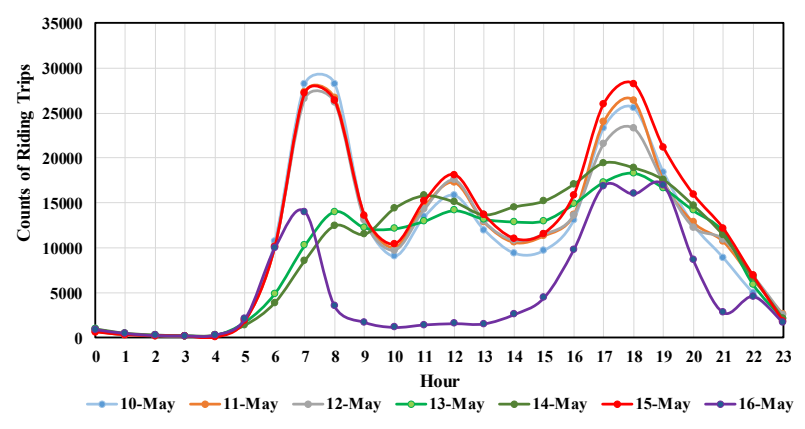

Figure 1. Temporal characteristics of riding during a week

The use of the Mobike bicycles is significantly different on weekdays and weekends (Figure1). Figure 2 clearly shows a morning peak (7-9h) and evening peak (17-19h), corresponding with typical commute time. At noon, some users' dining activities triggered a certain close-distance riding behavior, which formed a noon peak. Different from the riding characteristics of the working days, there are many recreational and leisure riding behaviors on the weekends. The distribution of riding time is more balanced, and there is no obvious morning and evening peak phenomenon.

(2) Spatial characteristics of riding behavior 
The spatial distribution of riding behaviour varies with different roads (Figure 2) and people prefer to choose trunk roads for cycling trips. Spatial hotpot detecting method based on the kernel density is applied to identify the active degree of bike sharing trip during a whole weekday (Figure 3). The red colour represents a high active degree and the green and blue colour means the low degree. Note that almost no riding occurred in the early hours of the morning and late at night. The characteristics of three riding peaks are obvious in the figure. A large number of travels occurred in Second Ring to Fourth Ring Road, and some travel activities were concentrated near traffic sites.

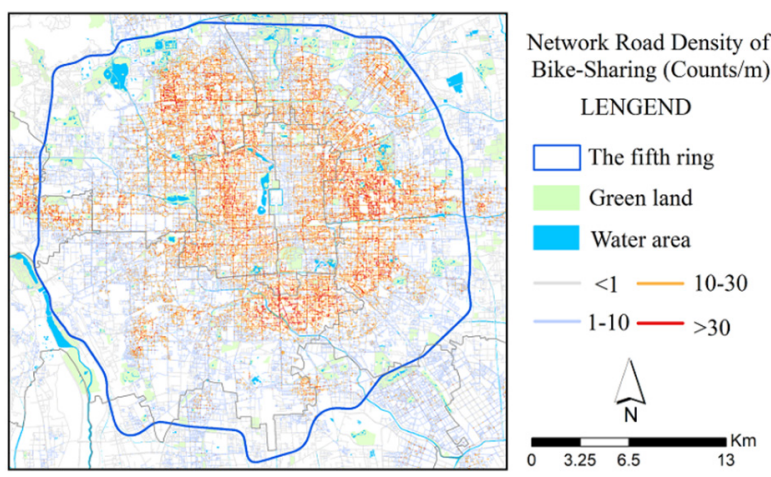

Figure 2. Network road density of shard bicycles

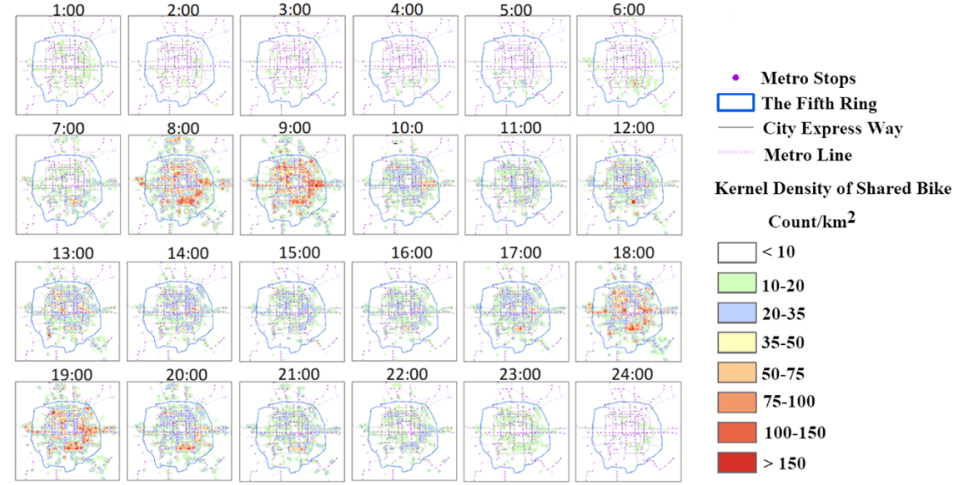

Figure 3. Kernel density of shared bike per hour a day

\section{(3) Patterns of riding behavior}

Different riding patterns happens in different space and change over the time at two scales of day and hour. During morning peak and evening peak on weekdays, more than 60 percent of riding trips are corresponding with typical commuting activities. The observed commuting pattern of morning peak (Figure 4(a) and (b)) implies that the majority of shared bicycle trips might relate to home, transports, commercial area and some institution. For example, students choose shared bicycles to do some school activities, people prefer to use shared bicycles as a connection tool to bus station and metro stops and people handle daily affairs in some government agencies. However, a large part of the shared bicycle trips on weekends shows the characteristics of non-commuting riding pattern, which means more leisure activities take place at weekends (Figure 4(c) and (d)). Non-commuting pattern of riding behavior mainly occurs among residential areas, metro stops, bus stations and recreational facilities, such as parks, playgrounds, etc.

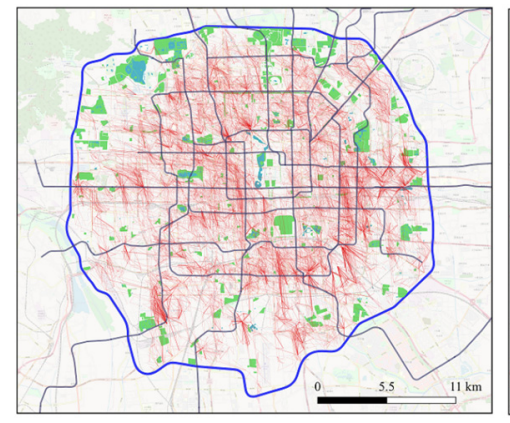

(a)Commuting pattern on weekday

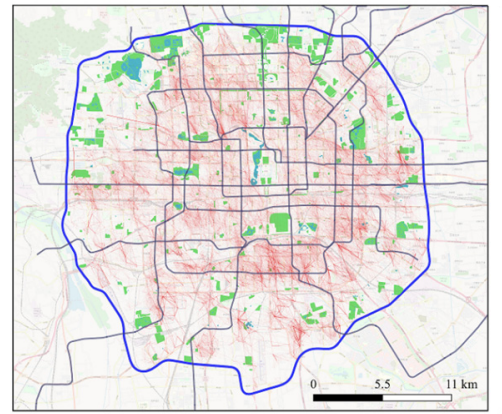

(c)Commuting pattern on weekend

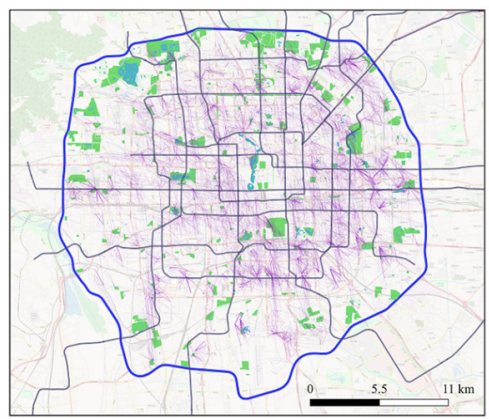

(b)Non-commuting pattern on weekday

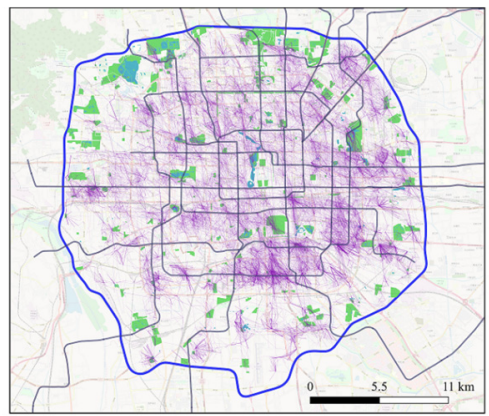

(d)Non-commuting pattern on weekend

\section{LENGEND}

OD flow of commuting pattern OD flow of noncommuting pattern

The fifth ring

Green land

Water area

Metro line

Figure 4. Spatial distribution of the shared bicycle OD flow 